\title{
Correspondence
}

\section{Quantum effects in the gravitational field}

I agree with Chiara Marletto and Vlatko Vedral that we need to test quantum gravity experimentally (Nature 547, 156-158; 2017). However, the idea of measuring quantum superpositions of the gravitational field, as Richard Feynman described, is hardly new.

The problem is that, with current measurement precision, we can detect either the gravitational field of an object or its quantum properties, but not both. The gap is closing slowly - a main topic at the Experimental Search for Quantum Gravity meeting last year in Frankfurt, Germany. This conference series, which, as Marletto and Vedral advocate, "bring[s] together the quantum- and gravity-physics communities, as well as theorists and experimentalists", has in fact been running since 2007 .

Austrian physicist Markus Aspelmeyer and his group have published promising work in this area (see, for instance, J. Schmöle et al. Class. Quantum Grav. 33, 125031; 2016). Several independent teams are studying whether it is possible to distinguish classical from quantum correlations in the cosmic microwave background. Others are trying to quantify whether quantum entanglement that is mediated by gravity can be used to pin down the quantum nature of space and time. (For a summary, see also go.nature.com/2woh6sz.) Sabine Hossenfelder Frankfurt Institute for Advanced Studies, Frankfurt am Main, Germany. hossi@fias.uni-frankfurt.de

Marletto and Vedral reply - We proposed that quantum effects in the gravitational field could be witnessed by probing the field using two masses, with each one in a superposition of two locations (Nature 547, 156-158; 2017). Earlier proposals, from Richard Feynman and from
Markus Aspelmeyer's group, instead use a single superposed mass and a classical test mass as a probe.

Our proposal depends on detecting entanglement between the two masses, generated by gravitational interaction (C. Marletto and V. Vedral Preprint at http:// arxiv.org/abs/1707.06036; 2017). A related proposal has emerged from the quantuminformation community (S. Bose et al. Preprint at http://arxiv. org/abs/1707.06050; 2017). More-detailed predictions, based on current quantum-gravity approaches, are needed for these schemes. We would therefore welcome an interdisciplinary meeting involving the communities that work on quantum information, quantum foundations and quantum gravity. Chiara Marletto, Vlatko Vedral University of Oxford, UK. chiara.marletto@physics.ox.ac.uk

\section{Venezuela's botanic heritage in jeopardy}

The National Herbarium of Venezuela, a cornerstone institution for plant sciences in Latin America, is under threat from vandals. We implore the botanical community to join our efforts to save it.

The vandalism is a result of Venezuela's fragile economic and political situation, and has led the research institute at the Caracas Botanical Garden (the IEJB), which hosts the herbarium, to suspend access to it. The institute's electrical and computing facilities have been repeatedly plundered this year, wiping out databases and compromising the herbarium's collection of more than 400,000 specimens.

Herbaria enable the study and classification of Earth's biodiversity, promote the discovery of species and provide DNA samples for analysis of extant and extinct populations. Among other services, such collections catalogue past and present plant distributions, establish the onset of plant invasions, and record alterations in plant seasonal cycles caused by climate change.

Civil society and the Venezuelan authorities must protect this priceless resource for science and the cultural good. The Latin American Plant Sciences Network offers a channel for the aid urgently needed for its rescue (e-mail: rlb@iecologia.unam.mx). Juan Núñez-Farfán, Javier A. Simonetti Latin American Plant Sciences Network, Santiago, Chile. Thalia Morales Roja National Herbarium of Venezuela, Caracas. farfan@unam.mx

\section{Testers wanted for article search tool}

We invite the scientific community to test a search engine we have developed for the biomedical literature (see http://pubmed.ict.griffith.edu.au). The aim of this Article-based PubMed Search Engine is to find only those publications that are most relevant to any particular article.

By extracting keywords from a paper's title and abstract, the search engine reveals every related study indexed in PubMed up to 26 July 2017 — typically as many as 60 publications per paper. We then refine the results by asking researchers to score them for relevance, which takes just a few minutes. All such evaluations will be curated into a benchmark data set that can be downloaded and distributed for free.

The web server has attracted several thousand visitors from more than 50 countries since its launch in July. Preliminary data indicate an overall success rate of $80 \%$ in identifying relevant articles.

Peter Brown, Yaoqi Zhou Griffith University, Queensland, Australia.

yaoqi.zhou@griffith.edu.au

\section{Fertile mind that led to unexpected places}

The late David Jones, who contributed to Nature under the pseudonym Daedalus, produced an innovative idea every week - and not just in his own field (see go.nature.com/2x5n3ne). From 1988 to 2000, he proposed ingenious 'inventions' that ranged from calming children with amniotic fluid to refolding prion proteins with heavy water to treat bovine spongiform encephalopathy (see Nature 390, 126-127; 1997).

Although his published ideas incorporated deliberate flaws, about one in five of them turned out to be viable (see also The Inventions of Daedalus (W. H. Freeman, 1982) and The Further Inventions of Daedalus (Oxford Univ. Press, 1999)).

A physical chemist, he was a pioneer in the use of mass media for research. For example, he debunked the theory that Napoleon was deliberately poisoned by arsenic after he speculated in a radio broadcast that wallpaper in Napoleon's St Helena home contained the poison. This unearthed a sample of the original wallpaper that satisfactorily accounted for the levels of arsenic detected in the emperor's hair (D. E. H. Jones and K. W. D. Ledingham Nature 299, 626-627; 1982).

Jones also wrote two books on the origin of innovative ideas (The Aha! Moment Johns Hopkins Univ. Press, 2012; and Why Are We Conscious? Pan Stanford, 2017). The second was published days before he died on 19 July at the age of 79 .

Daedalus eschewed hot topics, recognizing that exciting ideas are often to be found in ignored areas. His approach to science holds important lessons for everyone struggling to survive in our metrics-driven world.

Martyn Poliakoff University of Nottingham, UK.

Robin Perutz University of York, UK.

martyn@poliakoff.org.uk 